\title{
Si livellano gli investimenti azionari al saggio di interesse di mercato?
}

\author{
Memoria ai Lutgr AMoroso (u Roma)
}

Ad Enrico Bompiani in occasione del suo givbileo scientifico.

Sunto. - 1. Il tema del presente articolo - 2, $\dot{E}$ vincolato alla ipotesi della concor. renza perfetta - 3. Il saggio di interesse di mercato - 4. La realtì che si cela sotto il velo monetario - 5. Il divario fra saggio di mercato e saggio di capitalizzazione rap. presenta una speranza matematica - 6. Come si calcola questa speranza per $i$ titoli azionari. 7. La formulazione generale del principio marginale nel settore degli inve. stimenti.

1. Il tema che mi propongo di trattare nasce dal contrasto fra una tesi ed un'antitesi, come è qui indicato:

Thsr. - 亡̀ canone dell' economia classica che il risparmio si distribuisce tra $\mathrm{i}$ diversi investimenti in modo da livellare al margine tutti $\mathrm{i}$ rendimenti, arrestandoli ad una quota comune, che è quella rappresentata dal saggio di interesse di mercato. Dal che discende, come corollario, che, appunto perche livella i corrispondenti investimenti, il saggio di mercato lega fra di loro tutti i prezzi, per esempio il prezzo della carne con quello dell' acciaio, il prezzo dei medicinali con quello dei tessuti, il prezzo delle automobili con quello delle macchine agricole e così via. Sicchè il saggio di interesse può essere considerato come il centro di gravita, il baricentro, verso cui gravitano tutti i prezzi.

ANTitesi. - Viceversa chi esamina i listini di borsa e paragona dividendi e quotazioni, trova saggi di rendimento, che sono diversi da titolo a titolo e generalmente diversi da quello ehe nel gionno è il saggio di mercato. E questa rilevazione costituisce una manifesta contradizione calla proposizione della economia classica; sopra indicata.

Che cosa dobbiamo allora coneludere? Che la contradizione è reale ed inconciliabile e quindi la proposizione classica deve essere respinta come erronea, perchè in contrasto coi fatti sperimentali ? Ovvero che la contradi. zione è solamente apparente, imputabile all'errore di calcolare il saggio di inve. 
stimento, basandosi unicamente sui frutti presenti, senza considerare i frutti sperati in futuro? Il che significa poi considerare solo il reddito attuale e non l'incremento di valore del capitale, derivante dall' incremento presunto dei redditi futuri.

Dimostrerò qui che il dilemma si presenta non solo per i valori azionari, ma per tutte le forme di investimento: che la contradizione è solo apparente: che la considerazione dei frutti futuri esprime una speranza matematica, dalla quale il mercato non prescinde; che nei confronti dei titoli azionari siffatta speranza matematica assume un valore preciso e ben determinato: che la formula che la rappresenta può essere considerata come espressione del principio marginaie, valida qualunque sia la natura e la forma dell'inve. stimento.

2. - La dimostrazione è tuttavia subordinata alla ipotesi che il mercato operi in condizioni di concorrenza perfetta. Questo vuol dire che il sistema dei prezzi possa essere raffigurato come una costellazione siderea, che guida e non è guidata. Tutti gli operatori guardano al movimento dei prezzi, regolano su di essi ia loro condotta, ma nessuno ha la possibilità di influire direttamente sul loro movimento. Quindi esclusione non solo di ogni forma di coa. lizione e di monopolio, ma altresi di ogni privilegio, quale sarebbe, per esempio, quello che deriverebbe naturalmente ad un operatore, che avesse la possibilita di presentare sul mercato una quota rilevante della domanda o della offerta collettiva. Una configurazione di questo genere si presenta approssimativamente neila agricoltura, dove nessun latifondista potrebbe essere cosi temerario da affermare che egli, allargando o restringendo la sua produzione, potrebbe direttamente influire sul prezzo del grano; può pure presentarsi, sempre approssimativamente, nella industria tessile, non si presenta generalmente nella industria chimica e tanto meno nell' industria siderurgica e nell'industria meccanica ed automobilista.

Richiamo l'attenzione sugli avverbi "direttamente,, ed "approssimativamente,. Essi stanno ad indicare che ogni operatore, qualunque sia la sua quota, esercita sempre una influenza sul prezzo. Anche se i coltivatori di grano fossero, ad esempio, 10 milioni e ciascuno portasse sul mercato esattamente un decimilionesimo della quota totale, la influenza che il singolo operatore eserciterebbe sul prezzo sarebbe piccolissima, ma non nulla. Il nulla si avrebbe come caso limite solo nella ipotesi che il numero dei produttori fosse infinitamente grande e la quota di ciascuno infinitamente piccola. Ma l'infinitamente grande e l'infinitamente piccolo sono astrazioni matematiche, che non hanno riscontro nella realtà. Per questo la configurazione della perfettta concorrenza può essere un modello teorico, un caso limite, 
una ipotesi di studio; ma è proprio questa ipotesi di studio, che ci ha fatto un'anima capace di orientarsi nel groviglio dei fatti concreti.

3. - Che cosa è il saggio di interesse di mercato?

E - rispondo - un termine convenzionale, che è necessario introdurre perchè le operazioni finanziarie (di oredito) ohe vengono eseguite in uno stesso mercato, in uno stesso momento, sono stipulate a tassi diversi, secondo la loro specifica natura. La convenzione può riferirsi o ad un determinato titolo di Stato, o ad una media dei titoli di Stato e dei titoli garantiti dallo Stato o ad una media di tutti i titoli a reddito fisso e le quattro alternative portano a risultati praticamente convergenti. Ma poichè la seconda la terza e la quarta alternativa esigerebbero una ulteriore precisazione circa la media da adottare (aritmetica, geometrica, armonica, etc.) e circa il peso da attribuire a ciaseun termine, preferisco qui attenermi alla prima alternativa.

Assumo quindi come saggio di mercato il saggio di capitalizzazione del " consolidato $5 \%$,, quale risulta quotidianamente dalla quotazione allá Borsa di Milano. Al 29 settembre 1961 p. e. questa quotazione era di lire 105,50 e poichè nei dodici mesi precedenti il titolo aveva fruttato 5 lire, in due cedole di lire 2,50 ciascuna, messe a pagamento la prima il $1^{\circ}$ gennaio e l'altra il $1^{\circ}$ luglio, il gioco degli interessi per le frazioni di anno era di 9 mesi per la prima cedola e di 3 mesi per la seconda, onde detto $i$ il saggio di mercato risulta l'equazione:

$$
i=\frac{5+2,50 i}{105,50}
$$

dalla quale si ricava $i=0,0485$. Questa cifra rappresenta secondo la convenzione adottata il saggio di mercato, sulla piazza di Milano, il giono 29 settembre 1961 .

4. - Nessuno meglio di Einaudi ha squaroiato il velo monetario che impedisce di scorgere la viva realtà economica, che nasce dalla combinazione del presente e del futuro. In un Saggio pubblicato nel 1938 nella Rivista di Storia Economica (pagine 166-174), che reca il titolo " $I$ pazzi ed $i$ savi nella costruzione della terra italiana, egli ricorda l'opera dei mercanti senesi, pisani, lucchesi, i quali dal 1200 in poi seguitarono ad investire nella terra $i$ guadagni tratti dai fondaci, costruendo ville, livellando terreni, piantando olivi, coronando le vette di cipressi ed ingentilendo con piante da frutta e da orna. mento $i$ poderi. Opera analoga a quella compiuta dai mercanti milanesi, quando, tra il duecento ed il cinquecento e poi di nuovo nel settecento, spianavano terreni, colmavano bassifondi, derivavano canali e roggie, dotava- 
no di caseggiati monumentali acquitrini e brughiere. E si domanda: Ragio. nava questa gente ? Certo sragionava - risponde - perchè investiva i risparmi all' uno e forse meno per cento, quando ad essi si offrivano investimenti, ritenuti allora ugualmente sicuri, al 4 e al 5 per cento. Alla stessa stregua sragionano oggi tanti altri pazzi economici, i quali vanno migliorando, costruendo e bonificando dappertutto a tassi di frutto i quali, quando la mèta sia toccata, si aggireranno forse fra lo zero ed il due per cento dei capitali investiti.

Se non ci fossero stati questi pazzi, la terra italiana non sarebbe oggi quella che è. "Questa è - Einaudi conclude - la verità fondamentale nella storia dell'agricoltura italiana,., E poi si domanda: "Ma questi pazzi erano proprio pazzi ?,".

La risposta è trionfale: "Forse che industriali e commercianti - egli scrive - lavorano per disperdere i frutti del loro lavoro? E come conserverebbero, se non investissero nella terra? Si conoscono forse investimenti atti a resistere alle guerre, alle rivoluzioni, alle variazioni monetarie, meglio degli investimenti terrieri? Resistono male tutti; ma quale resiste meno peggio? L' oro, le gemme, i quadri di autore, i libri preziosi, i sopramobili rari, per il piccolo spazio tenuto e la facile invisibilita, resisterebbero egregiamente, ma il possesso di oro e di gemme diventa, a tratti di secoli, reato; ma le quadrerie, le biblioteche ed i ricordi di famiglia si disperdono, per la difficoltà della conservazione, per ignoranza e noncuranza. Se la terra, pur aperta alla grandine ed agli uccelli da preda, è dunque fornita di un grado di resistenza maggiore dei valori mobiliari, dei depositi bancari, degli impianti industriali e dei fondi di commercio, noi forse dobbiamo abituarci a considerare $11 \%$ terriero uguale al $5 \%$ mobiliare.

$L$ 'istinto terriero che parifica l'nno al cinque è forse un inconsapevole ricordo ancestrale, che tiene conto degli imponderabili ignorati dalla logica.

Ed allora chi sono i pazzi e chi sono i savi?,

5. - La geniale intuizione di Einaudi vale non solu per gli investimenti fondiari, ma vale in generale per tutti gli investimenti, che non hanno carattere prettamente monetario. Il valore del capitale (l'immobile, la fabbrica, l'emporio commerciale) si ragguaglia al valore attuale di tutti i redditi futuri, quali attualmente son presunti dal mercato, e scontati al saggio di mercato.

$\dot{\mathrm{E}}$ il principio della capitalizzazione dei redditi, dal quale discende che il valor capitale varia da istante ad istante, anche se la variazione è latente e si manifesta visibilmente solo all'atto della vendita o comunque all'atto di un trapasso di proprieta. Il saggio annuo di capitalizzazione è in ogni istante il rapporto fra il reddito realizzato nell'anno precedente ed il valore del capitale nell'istante. In genere fra qusto saggio ed il saggio di interesse di mercato corre un divario, che rende alternativamente l'uno all'altro superiore. E l'alternativa dipende dalla previsione del mercato oirca il flusso dei red- 
diti futuri. Precisamente il saggio di capitalizzazione è inferiore al saggio di mercato, quando il mercato presume un reddito futuro crescente, superiore nell' ipotesi opposta.

Cosî - ritornando alla analisi di Einaudi - il maggior grado di resistenza attribuito agli investimenti fondiari, viene ad identificarsi con la previsione di uno svolgimento orescente del reddito futuro, che fornisce i mezzi occorrenti per la resistenza. Tipico è il caso della difesa contro le svalutazioni monetarie, della quale è esempio luminoso il rapido adeguamento del prezzo dei prodotti agricoli (cioè del reddito) e conseguentemente del valore capitale delle terre nel primo e nel secondo dopoguerra (1918.22, 1945.50).

Viceversa il rovesciamento del divario, che si manifesta quando il saggio di capitalizzazione è superiore al saggio di mercato, indica la previsione di uno svolgimento decrescente del reddito futuro. Così avviene, per esempio, per una miniera, quando si manifestano i sintomi di un prossimo esaurimento o per una fabbrica, quando appare lo spettro di una flessione della domanda. In tal modo il divario fra il saggio di mercato ed il saggio di capitalizzazione, se è quantità positiva. riflette una speranza matematica e nella ipotesi opposta riflette un timore, cioè una speranza negativa. L'aggettivo matematica sta ad indicare che è in gioco un rudimentale calcolo de probabilità, in relazione alla previsione dello svolgimento del reddito futuro.

Formalmente il termine "futuro,, corrisponde alla successione indefinita dei tempi. In realtà si riferisce essenzialmente al futuro prossimo, per il fatto che nel calcolo finanziario il futaro remoto rapidamente impallidisce. Al saggio del $5 \%$, che è il valore centrale del saggio di mereato, il valore attuale di una rendita annua posticipata di 100 temporanea per 20 anni, è 12,46 ; il valore di una rendita temporanea per 40 anni è 17,16 ; quello di una rendita perpetua è 20 e questo significa che i primi 20 anni equivalgono sotto l'aspetto finanziario al $62,30 \%$ di tutto il futuro; i primi 40 anni equi. valgono ad $85,80 \%$.

6. - La correlazione fra il saggio di capitalizzazione e lo svolgimento presunto del reddito futuro, che per il fondo rustico, per l'immobile, per la fabbrica, l'emporio è il risultato di una felice intuizione, assume per i titoli azionari il carattere semplice ed elegante di un teorema matematico.

Detto:

$t$ il tempo, assunto come origine l'istante attuale, verso positivo dal presente al futuro, unità di misura l'anno;

$i$ il saggio di interesse di mercato all' istante $t=0$;

$Q$ la quotazione di borsa all' istante $t=0$;

$D$ il dividendo distribuito nell' altimo esercizio, chiuso prima dell'istante $t=0$

Dhit)dt il flusso del reddito futuro, presunto dal mercato nel tempu* 
scolo $d t$, compreso fra $t$ e $t+d t$ (con che viene implicitamente a porsi $h=1$, per $t=0$, il principio della capitalizzazione dei redditi si esprime con la formuia:

$$
Q=D \int_{0}^{\infty} e^{-i t} h(t) d t
$$

In questa formula $Q, D, i$ rappresentano rispettivamente la quotazione del titolo, il dividendo dell' ultimo esercivio, il saggio di mercato, tutte e tre le quantità essendo considerate all' istante $t=0$. Così, nella formula, $D$ ed $i$ appaiono come costanti, $Q$ come funzione di $D$ e di $i$ e come fanzionale nei confronti di $h(t)$. Precisamente la formula (1) esprime che la quotazione di borsa, considerata come funzione del saggio di mercato, è la trasformata $d i$ Laplace della funzione che rappresenta lo svolgimento del reddito futuro. Nei termini propri di questa trasformazione, il reddito futuro presunto è l'originale, la quotazione di borsa la sua immagine.

Ma due titoli, ai quali corrispondono nello stesso istante gli stessi valori del dividendo $D$ e della quotazione $Q$ sono finanziariamente equivalenti. Pos. siamo quindi, senza alterare la generalità, dare alla funzione $h(t)$ la forma esponenziale

$$
h(t)=e^{p t}
$$

nella quale l'esponente $p_{2}$. qui pensato indipendente da $t$, è tale da verificare alla (1). Segue allora

$$
Q=D \int_{0}^{\infty} e^{-(i-p) t} d t
$$

ed eseguendo la integrazione, supposto $p<i$ :

$$
Q=\frac{D}{i-p}
$$

da cui, detto $j$ il saggio di capitalizzazione, si ricava

$$
j=i-p
$$

L'esponenziale $e^{-p}$ indica così la ragione di nua progressione geometrica, con eni si svolge un reddito futuro, finanziariamente equivalente al reddito previsto dal mercato. Il parametro $p$ rappresenta allora la speranza matema. tica, quale è nell' istante attuale $t=0$. La progressione può essere crescente o decrescente. Nella prima ipotesi $p$ è quantità positiva e rappresenta una vera e propria speranza. Nella ipotesi opposta (progressione decrescente) $p$ è quantità negativa, e rappresenta un timore. 
Nella prima ipotesi $(p<0)$, in cui il reddito futuro è presunto crescente, il valore aritmetico di $p$ è vincolato alla condizione di essere inferiore al saggio di mercato $i$. Se così non fosse, l'integrale di cui alla formula (3) sarebbe divergente e questo significherebbe che nessuna somma di denaro, per quanto fosse elevata, sarebbe sufficiente a comprare il titolo. Evidentemente il mercato non può fare una previsione del genere.

Nessun vincolo aritmetico sussiste invece nella ipotesi opposta, in cui per essere il reddito futuro presunto decrescente, $p$ è quantită negativa. Al crescere del valore assoluto di $p$, la quotazione $Q$ diminuisce, tendendo al limite allo zero e siffatta tendenza non è in contrasto colla realtà sperimentale, la quale non esclude che il capitale sociale possa essere interamente perduto. L'eventualità di perdite, che ecedono il capitale sociale non si riflette sul titolo, in quanto il suo possessore, cioè l'azionista, non è, per legge, tenuto a risponderne. Colla disposizione di legge concorda la formula (3), che esclude che $Q$ possa avere un valore negativo.

7. - La formula (5) esprime il principio marginale nel settore degli investimenti e dimostra che il saggio di mercato non è solo il centro di gravità di tutti i prezzi attuali, ma è altresì il parametro, che lega il presente al futuro.

Qualunque sia la forma conereta dell' investimento (il fondo rustico, l' immobile, la fabbrica, il pacchetto azionario), il mercato quota alto, quando la speranza matematica è quantita positiva (reddito futuro presunto crescente); basso nella ipotesi opposta.

Siffatte quotazioni variano di giorno in giorno, riflettendo ogni giorno lo stato d'animo, le speranze, i timori, le angustie, le preoccupazioni di quella quota parte degli operatori che in quel giorno comprano o vendono. E le continne oscillazioni dei titoli esprimono appunto che lo stato d'animo degli operatori che sono oggi presenti sul mercato, è diverso da quello degli operatori che erano presenti ieri e da quello degli operatori che saranno presenti domani.

Ma per il fondo rustico, per l'immobile, per le ditte industriali e com. merciali, le fluttuazioni gionnaliere restano ignote o per lo meno solo parzialmente note alla grande massa del pubblico. Sono invece palesi per i titoli azio. nari, per i quali, anche in condizioni di concorrenza, affiorano quotidianamente tutte le debolezze umane, delle quali il mercato è per così dire il totalizza. tore. Le continue fluttazioni dei corsi ne sono la manifestazione visibile. Che se poi la concorrenza viene meno per la pressione di manovre dirette al rialzo o al ribasso, la borsa diventa un mare in burrasca, nel quale le tendenze che sono espressione del calcolo economico dei singoli operatori, vengono sopraffatte da altre tendenze, che possono aver origine in manovre speculative o comunque rappresentare aspirazioni, giudizi, ideologie, aventi carattere politico. 